\title{
A Quality Improvement Initiative to Decrease Maternal Morbidity in Placenta Accreta Spectrum with a Standardized Approach: A Single Institution Experience
}

\author{
Ivy L Lersten*, Christina E Rodriguez, Jamie R Gilroy, Dina M Fink and Saketh R Guntupalli \\ Department of Obstetrics and Gynecology, University of Colorado School of Medicine, Aurora, \\ Colorado, United States
}

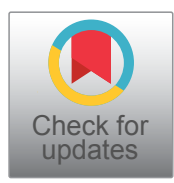

\begin{abstract}
Placenta accreta spectrum (PAS) is a rare, though increasing complication of pregnancy. Prior studies have suggested standardized management to PAS at tertiary centers can improve outcomes. We studied the effect of standardized multidisciplinary care at a single tertiary care center on composite maternal morbidity by reviewing outcomes retrospectively before and after implementation of a standardized treatment protocol. We found no statistical difference in composite outcomes in pre- and post-implementation groups though there was a lower likelihood of emergent deliveries, smaller amount of blood loss and decreased rate of emergent delivery. A standardized approach to PAS care may lead to improvements in some maternal outcomes.
\end{abstract}

\section{Introduction}

Placenta accreta spectrum (PAS) refers to abnormal placentation disorders including placenta accreta, increta and percreta. The diagnosis can be made clinically by the inability to separate the placenta from the uterus in the third stage of labor, or it can be diagnosed on pathology. If the diagnosis is not suspected prior to delivery and there is an attempt to deliver the placenta, there can be severe maternal complications from catastrophic bleeding. Even when it is suspected, there are significantly increased maternal risks including excessive blood loss, need for large volume blood transfusion, surrounding organ damage during surgery, intensive care unit (ICU) admission, need for reoperation, venous thromboembolism, and death [1,2]. Additionally, there are risks to the fetus, mostly related to the necessity of preterm delivery.

The incidence of PAS is on the rise which mirrors the rise in cesarean delivery rates across the country. A recent estimate of the rate of placenta accreta spectrum was $1 / 533$ births compared with $1 / 7000$ in the 1970 s [2,3]. The major risk factors for placenta accreta spectrum are prior cesarean delivery or other uterine surgery, and presence of placenta previa [1,4]. The American College of Obstetrics and Gynecology has put a strong emphasis on decreasing the rates of primary cesarean delivery in an effort to decrease the risks of downstream complications, however, the United States cesarean delivery rate was still $32 \%$ in 2018 [5].

Appropriate identification of PAS is critical to ensure proper referral and preoperative preparation. Diagnosis is usually accomplished by ultrasound with or without the aid of mag- netic resonance imaging (MRI) $[6,7]$. Antenatal diagnosis allows for organization of a team of expert physicians and personnel that are experienced with complex cases, as well as planning for a controlled delivery. The most accepted mode of management is cesarean hysterectomy, although some groups advocate for a conservative approach through uterine preserving surgery, methotrexate therapy, or uterine artery occlusion [8,9].

While increasing in frequency, PAS remains a somewhat rare occurrence for each individual institution. In recent years, studies demonstrated that there are better outcomes when placenta accreta spectrum cases are managed in tertiary care centers with a multidisciplinary approach $[10,11]$. The center must have the ability to perform accurate prenatal identification of cases as well as be able to perform massive transfusion during a surgery. Additionally, there must be intensive care units for adults and neonates.

Our objective was to evaluate the effect of a standardized

*Corresponding author: Ivy L Lersten, Department of Obstetrics and Gynecology, University of Colorado School of Medicine, Aurora, Colorado, USA

Accepted: January 21, 2021

Published online: January 23, 2021

Citation: Lersten IL, Rodriguez CE, Gilroy JR, et al. (2021) A Quality Improvement Initiative to Decrease Maternal Morbidity in Placenta Accreta Spectrum with a Standardized Approach: A Single Institution Experience. Annals Gynecol Obstet 5(1):105-109 
Citation: Lersten IL, Rodriguez CE, Gilroy JR, et al. (2021) A Quality Improvement Initiative to Decrease Maternal Morbidity in Placenta Accreta Spectrum with a Standardized Approach: A Single Institution Experience. Annals Gynecol Obstet 5(1):105-109

multidisciplinary approach to PAS management on composite maternal morbidity and mortality at a tertiary care center. We hypothesized that a standardized approach would decrease maternal morbidity without an increase in adverse effect on short-term neonatal outcomes.

\section{Materials and Methods}

This is a before-after retrospective cohort study at a single academic center. The study was approved by the Colorado Multiple Institutional Review Board. A quality improvement initiative was instituted in January 2013 to standardize the management of placenta accreta spectrum disorders. The protocol included coordinated care between specialists in maternal fetal medicine, gynecologic oncology, anesthesiology, urology, interventional radiology, and neonatology. Each patient with suspected PAS was evaluated with ultrasound and MRI imaging, and a multidisciplinary care conference was held to plan for delivery and review imaging. For each case, the surgical approach was determined, and timing of delivery was scheduled between 34 and 36 weeks. Decision to place ureteral stents was made depending on location and degree of placental invasion on MRI. Outpatient care was primarily managed through maternal fetal medicine physicians and outpatient consultation with a gynecologic oncologist was standard. Women were admitted the night prior to planned surgery, if not already inpatient on the antepartum service.

At time of surgery, blood products were cross matched including 6 units of packed red blood cells, 6 units of fresh frozen plasma and 1 unit of platelets. The surgery occurred in a hybrid operating room which allowed easier access to interventional radiology procedures, whether or not hypogastric artery balloon placement was part of the planned surgical approach. The obstetrical anesthesiology team placed an arterial line and at least two large bore intravenous lines. Anesthesia was provided via combined spinal epidural for the neonatal delivery followed by general anesthesia for the hysterectomy portion.

Ureteral stents and prophylactic hypogastric artery balloons were utilized on a case-by-case basis depending on the preoperative imaging. The maternal fetal medicine team, consisting of a maternal fetal medicine attending physician, maternal fetal medicine fellow and physician, started the surgery with a midline vertical incision and sonographic mapping of the placental edge to plan a safe location for hysterotomy. After delivery of the infant, the cord was tied off and replaced inside the uterus without attempted removal of the placenta. The hysterotomy was then closed and the hysterectomy portion of the procedure was performed by the gynecologic oncology team which consisted of a gynecologic oncology attending, a gynecologic oncology fellow and a resident physician. A total hysterectomy was performed with identification of ureters, and ureterolysis was performed when necessary to ascertain their course. Pedicles were controlled with a combination of standard suture ligation and the Ligasure device. Bilateral salpingectomy was performed if desired by the patient. A single intra-abdominal Jackson-Pratt drain was typically placed. Postoperatively, the patient was taken directly to the surgical ICU for one night of observation unless the in- traoperative team determined they were stable for postpartum floor care.

All women who delivered after January 1, 2013 through January 2017 with suspected PAS were in the standardized management arm including women who were transported in urgently without the benefit of an outpatient workup and care conference. All women who delivered between January 1, 2007 and December 31, 2012 were in the historic comparison group. Historically, suspected PAS was managed based on primary general obstetrician attending preference with consultation to gynecologic oncology and other services at their own discretion.

Clinical data was collected retrospectively by chart review. The maternal clinical parameters that were extracted included demographic factors, surgical history, ultrasound and MRI imaging characteristics, intraoperative use of ureteral stenting, hypogastric artery balloon placement, type of anesthesia, operative time, estimated blood loss (EBL), blood product transfusion, intraoperative and postoperative complications, ICU length of stay, post-operative hospital length of stay, readmission, reoperation, and death. Neonatal outcomes were also collected including birth weight, Apgar scores, transient tachypnea of the newborn, respiratory distress syndrome, intraventricular hemorrhage, and death.

The primary outcome was a composite of maternal morbidity and mortality measures including ICU stay $\geq$ two days, blood transfusion $>4$ units, reoperation within six weeks of surgery, readmission within 30 days, infectious complications, or hospital length of stay > five days after surgery. Secondary outcomes included whether delivery was scheduled or urgent, gestational age at delivery, EBL, neonatal birth weight, and neonatal respiratory distress syndrome.

Categorical data was presented as numbers. Comparison between groups was performed with chi-square test and t-test. A $P$ value of $<0.05$ was considered statistically significant. The effect estimates were calculated as odd ratios (ORs) with a $95 \% \mathrm{Cl}$. Statistical analysis was performed with the use of SPSS 25.0 .

\section{Results}

Through our study period, 71 cases of invasive placentation were identified. This included 29 cases prior to implementation of standardized care and 42 cases after. The pre- and post-implementation cases had similar age, gravidity, parity and underlying chronic medical conditions such as chronic hypertension or pre-gestational diabetes (Table 1). The post-implementation group was more likely to have a concurrent placenta previa diagnosis (OR 3.79, 95\% Cl 1.13-12.69) and MRI imaging during the diagnostic evaluation (OR 3.65, $95 \% \mathrm{Cl} 1.35-9.88)$. Emergent deliveries were less likely during the post-implementation period (OR $0.32,95 \% \mathrm{Cl} 0.12-0.85$ ). The most common indications for emergent delivery included vaginal bleeding, preterm labor and preterm premature of membranes. Overall, there was no statistical difference in mean gestational age at time of delivery between pre- and post-implementation 123 groups ( 35.38 c 3.23 weeks versus $33.69 \pm 3.91$ weeks, $P=0.06)$. 
Citation: Lersten IL, Rodriguez CE, Gilroy JR, et al. (2021) A Quality Improvement Initiative to Decrease Maternal Morbidity in Placenta Accreta Spectrum with a Standardized Approach: A Single Institution Experience. Annals Gynecol Obstet 5(1):105-109

Table 1: Demographics and patient characteristics.

\begin{tabular}{|c|c|c|c|c|}
\hline Characteristic & $\begin{array}{l}\text { Post- implementation } \\
(\mathrm{N}=42)\end{array}$ & $\begin{array}{l}\text { Pre- implementation } \\
(N=29)\end{array}$ & Odds ratio $(95 \% \mathrm{Cl})$ & $\boldsymbol{P}$ \\
\hline Age (y) & $33.05( \pm 5.69)$ & $33.41( \pm 4.92)$ & $\mathrm{n} / \mathrm{a}$ & 0.779 \\
\hline Gestational age at delivery (weeks) & $33.69( \pm 3.91)$ & $35.38( \pm 3.23)$ & $\mathrm{n} / \mathrm{a}$ & 0.060 \\
\hline Mean gravidity & $4.19( \pm 3.00)$ & $4.24( \pm 1.96)$ & $\mathrm{n} / \mathrm{a}$ & 0.923 \\
\hline Mean parity & $1.76( \pm 1.06)$ & $1.69( \pm 1.20)$ & $\mathrm{n} / \mathrm{a}$ & 0.789 \\
\hline History of cesarean delivery & $38(90.5 \%)$ & $21(72.4 \%)$ & $3.62(0.98-13.46)$ & 0.055 \\
\hline Number of prior cesarean delivery & $1.79( \pm 1.12)$ & $1.21( \pm 1.05)$ & $\mathrm{n} / \mathrm{a}$ & 0.031 \\
\hline History of other uterine surgery & $13(31.0 \%)$ & $11(37.9 \%)$ & $0.73(0.27-1.98)$ & 0.541 \\
\hline Concurrent placenta previa & $36(85.7 \%)$ & $19(65.5 \%)$ & 3.79 (1.13-12.69) & 0.031 \\
\hline Placental location & & & & 0.823 \\
\hline Fundal & $2(4.9 \%)$ & $0(0 \%)$ & $\mathrm{n} / \mathrm{a}$ & \\
\hline Anterior/central & $30(73.2 \%)$ & $22(78.5 \%)$ & & \\
\hline Posterior & $5(12.2 \%)$ & $3(10.7 \%)$ & & \\
\hline Partial/Low-lying & $4(9.8 \%)$ & $3(10.7 \%)$ & & \\
\hline Antenatal MRI performed & $29(69.0 \%)$ & $11(37.9 \%)$ & $3.65(1.35-9.88)$ & 0.011 \\
\hline Antenatal accreta diagnosis & $36(85.7 \%)$ & $14(48.3 \%)$ & $6.43(2.08-19.91)$ & 0.001 \\
\hline Pregestational diabetes & $1(2.4 \%)$ & $1(3.4 \%)$ & $0.68(0.04-11.38)$ & 0.791 \\
\hline Chronic hypertension & $2(4.8 \%)$ & $2(6.9 \%)$ & $0.68(0.09-5.09)$ & 0.703 \\
\hline
\end{tabular}

Data is mean \pm standard deviation, or $n(\%)$ unless otherwise specified.

Table 2: Composite adverse maternal outcomes.

\begin{tabular}{|l|l|l|l|}
\hline & Post- implementation $\mathbf{( N = 4 2 )}$ & Pre- implementation (N = 29) & $P$ \\
\hline Number of adverse events & $0.71( \pm 0.99)$ & $0.89( \pm 0.88)$ & 0.443 \\
\hline Composite adverse outcomes & & & 0.092 \\
\hline$\geq 1$ & $19(45.2 \%)$ & $19(65.5 \%)$ & \\
\hline $\mathbf{0}$ & $23(54.8 \%)$ & $10(34.5 \%)$ & \\
\hline
\end{tabular}

Data is mean \pm standard deviation, or $n(\%)$ unless otherwise specified.

Overall adverse outcomes based on composite maternal morbidity and mortality were similar pre- and post- implementation ( $0.71 \pm 0.99$ vs. $0.89 \pm 0.88, P=0.443$, Table 2 ). The post-operative hemoglobin was significantly higher after standardization $(10.8 \pm 4.8$ vs. $13.6 \pm 6.5, P=0.039)$. There was no difference in average units of blood transfused ( $4.05 \pm$ 4.96 vs. $5.10 \pm 4.48, P=0.362$ ). Other adverse outcomes were similar between the two groups including length of ICU and hospital stay, bowel, ureteral or bladder injury. There was one maternal death in our pre-implementation group and zero in our post group. Maternal delivery characteristics are summarized in Table 3.

Neonatal outcomes were deemed adverse if five-minute Apgar score was less than seven or if other complications were present such as transient tachypnea of the newborn, respiratory distress syndrome, intraventricular hemorrhage or neonatal death. Protocol use was not associated with any statistical difference in neonatal complications (Table 4).

\section{Discussion}

Placenta accreta spectrum increases the morbidity and mortality of pregnancy due to higher rates of bladder, ureteral and bowel injuries along with higher blood loss. The increasing prevalence of cesarean deliveries will mean more pregnancies complicated by PAS. Measures to improve maternal and neonatal outcomes are paramount. Prior studies have demonstrated improved outcomes amongst women who deliver at multi-disciplinary tertiary centers versus standardized obstetrical centers [10]. To further optimize delivery outcomes at a tertiary center, we demonstrate that the use of a standardized protocol resulted in a lower rate of emergent deliveries without lowering the gestational age of delivery. We found overall no difference in risk of composite adverse outcomes, though we suspect that could be due to our small sample size.

Advantages of a protocolized approach are a thorough, standardized predelivery evaluation of the extent of placental invasion. Our approach utilized the surgical expertise of gynecologic oncologists for the hysterectomy portion of the procedure. We believe that gynecologic oncologists' surgical experience helps to decrease length of operating time and thus, minimize intraoperative blood loss as evidenced by a 
Citation: Lersten IL, Rodriguez CE, Gilroy JR, et al. (2021) A Quality Improvement Initiative to Decrease Maternal Morbidity in Placenta Accreta Spectrum with a Standardized Approach: A Single Institution Experience. Annals Gynecol Obstet 5(1):105-109

Table 3: Maternal delivery characteristics.

\begin{tabular}{|c|c|c|c|c|}
\hline Characteristic & Post-implementation $(\mathrm{N}=42)$ & Pre-implementation $(\mathrm{N}=29)$ & Odds ratio $(95 \% \mathrm{Cl})$ & $\boldsymbol{P}$ \\
\hline $\mathrm{EBL}(\mathrm{cc})$ & $3026.19( \pm 2344.17)$ & $3412.07( \pm 1624.46)$ & $n / a$ & 0.445 \\
\hline Blood transfusion (units) & $4.05( \pm 4.96)$ & $5.10( \pm 4.48)$ & n/a & 0.362 \\
\hline $\begin{array}{l}\text { Pre and post operative hemoglobin } \\
\text { decrease (mean \% loss) }\end{array}$ & $10.8( \pm 4.8)$ & $13.6( \pm 6.5)$ & $n / a$ & 0.039 \\
\hline \multicolumn{5}{|l|}{ Ureteral stents placement } \\
\hline Yes & $13(31.0 \%)$ & $6(20.7 \%)$ & $1.72(0.56-5.22)$ & 0.340 \\
\hline No & $29(69.0 \%)$ & $23(79.3 \%)$ & & \\
\hline \multicolumn{5}{|l|}{ Hypogastric artery balloon placement } \\
\hline Yes & $8(19.0 \%)$ & $5(17.2 \%)$ & $1.13(0.33-3.88)$ & \\
\hline No & $34(81.0 \%)$ & $24(82.8 \%)$ & & 0.847 \\
\hline ICU LOS (days) & $0.62( \pm 0.85)$ & $0.50( \pm 0.79)$ & $n / a$ & 0.559 \\
\hline Hospital LOS (days) & $5.0( \pm 2.25)$ & $4.45( \pm 1.33)$ & $n / a$ & 0.241 \\
\hline Hysterectomy operative time (hrs) & $3: 21( \pm 1: 24)$ & $2: 58( \pm 1: 16)$ & $n / a$ & 0.269 \\
\hline \multicolumn{5}{|l|}{ Reoperation within 6 weeks } \\
\hline Yes & $3(7.1 \%)$ & $2(6.9 \%)$ & $1.04(0.16-6.64)$ & 0.968 \\
\hline No & 39 (92.9\%) & 27 (93.1\%) & & \\
\hline \multicolumn{5}{|l|}{ Readmission within 30 days } \\
\hline Yes & $2(4.8 \%)$ & $2(6.9 \%)$ & $0.68(0.09-5.09)$ & 0.703 \\
\hline No & $40(95.2 \%)$ & 27 (93.1\%) & & \\
\hline \multicolumn{5}{|l|}{ Infectious complication } \\
\hline Yes & $2(4.8 \%)$ & $2(6.9 \%)$ & $0.68(0.09-5.09)$ & 0.703 \\
\hline No & $40(95.2 \%)$ & 27 (93.1\%) & & \\
\hline \multicolumn{5}{|l|}{ Cystotomy } \\
\hline Yes & $3(7.1 \%)$ & $3(10.3 \%)$ & $0.67(0.12-3.56)$ & 0.635 \\
\hline No & $39(92.9 \%)$ & $26(89.7 \%)$ & & \\
\hline \multicolumn{5}{|l|}{ Ureteral injury } \\
\hline Yes & $1(2.4 \%)$ & $0(0 \%)$ & $n / a$ & \\
\hline No & 41 (97.6\%) & $29(100 \%)$ & & \\
\hline \multicolumn{5}{|l|}{ Bowel injury } \\
\hline Yes & $1(2.4 \%)$ & $1(3.4 \%)$ & $0.68(0.04-11.38)$ & 0.791 \\
\hline No & $41(97.6 \%)$ & $28(96.6 \%)$ & & \\
\hline \multicolumn{5}{|l|}{ Maternal death } \\
\hline Yes & $0(0 \%)$ & $1(3.4 \%)$ & $\mathrm{n} / \mathrm{a}$ & \\
\hline No & $42(100 \%)$ & $28(96.6 \%)$ & & \\
\hline
\end{tabular}

EBL: Estimated blood loss; cc: Cubic centimeter; LOS: Length of stay. Data are mean \pm standard deviation, or $\mathrm{n}$ (\%) unless otherwise specified.

Table 4: Neonatal outcomes.

\begin{tabular}{|l|l|l|l|l|}
\hline Characteristic & Post-implementation & Pre-implementation & Odds ratio (95\% Cl) & $\boldsymbol{P}$ \\
\hline Neonatal birthweight $(\mathrm{g})$ & $2297.51( \pm 533.93)$ & $2586.14( \pm 678.45)$ & n/a & 0.053 \\
\hline 5 minute Apgar $<7$ & $1(2.4 \%)$ & $2(6.9 \%)$ & $0.33(0.03-3.81)$ & 0.374 \\
\hline Transient tachypnea of the newborn & $3(7.1 \%)$ & $1(3.4 \%)$ & $2.15(0.21-21.8)$ & 0.516 \\
\hline Respiratory distress syndrome & $12(28.6 \%)$ & $11(37.9 \%)$ & $0.65(0.24-1.79)$ & 0.409 \\
\hline Intraventricular hemorrhage & $1(2.4 \%)$ & $0(0 \%)$ & n/a & $0.68(0.04-11.37)$ \\
\hline Neonatal death & $1(2.4 \%)$ & $1(3.4 \%)$ & 0.791 \\
\hline
\end{tabular}

Data are mean \pm standard deviation, or $\mathrm{n}(\%)$ unless otherwise specified. 
Citation: Lersten IL, Rodriguez CE, Gilroy JR, et al. (2021) A Quality Improvement Initiative to Decrease Maternal Morbidity in Placenta Accreta Spectrum with a Standardized Approach: A Single Institution Experience. Annals Gynecol Obstet 5(1):105-109

smaller post-surgical drop in blood counts after protocol implementation. In addition, more cases of placenta accreta spectrum were diagnosed antenatally after implementation which likely contributed to the lower rate of emergent deliveries. More predictable, controlled deliveries results in fewer errors, increased availability of essential operative staff and avoids anesthetic complications associated with emergent intubations. In addition, antenatal diagnosis allows for anticipatory guidance for delivery and neonatal counseling on the risks of preterm delivery. The treatment of placenta accreta spectrum leads to permanent sterilization for our patients, an outcome often challenging to endure, especially in emergent settings.

A key aspect of PAS management is balancing maternal morbidity with neonatal in utero growth. Our protocol was able to achieve our outcomes without worsening neonatal outcomes at the time of delivery. Multidisciplinary involvement in delivery timing was a critical part of our protocol especially with maternal-fetal medicine expertise to weigh the risks of preterm birth to help determine target gestational age of delivery.

Our study had several limitations. The rates of rare complications such as ureteral or bowel injury would require a much larger sample size than our study provided to identify significant differences. Our pre-standardization group was managed by individual physician preference - exact differences between prior management strategy and protocol management is unknown. In addition, as a tertiary care center, patients who were transported for emergent delivery with PAS did not have the benefit of standardized care.

In summary, the application of a standardized PAS protocol at a large tertiary academic center led to a lower rate of emergent deliveries without compromising neonatal outcomes. While overall adverse composite scores did not differ after standardization, we suspect this finding was limited by small sample size. Further studies of larger sample size are needed to investigate effects on rare cesarean hysterectomy complications in this setting. When possible, the workup and management of placenta accreta spectrum cases should be protocolized.

\section{Data Availability}

The data used to support the findings of this study are included within the article.

\section{Conflicts of Interest}

The author(s) declare(s) that there is no conflict of interest regarding the publication of this paper.

\section{Funding}

None.

\section{Acknowledgements}

None.

\section{References}

1. Silver RM, Landon MB, Rouse DJ, et al. (2006) National institute of child health and human development maternal-fetal medicine units network. Maternal morbidity associated with multiple repeat cesarean deliveries. Obstet Gynecol 107: 1226-1232.

2. Usta IM, Hobeika EM, Musa AA, et al. (2005) Placenta previa-accreta: Risk factors and complications. Am J Obstet Gynecol 193: 1045-1049.

3. Read JA, Cotton DB, Miller FC (1980) Placenta accreta: Changing clinical aspects and outcome. Obstet Gynecol 56: 31-34.

4. Bowman ZS, Eller AG, Bardsley TR, et al. (2014) Risk factors for placenta accreta: A large prospective cohort. Am J Perinatol 31: 799-804.

5. Martin JA, Hamilton BE, Osterman MJ, et al. (2019) Births: Final data for 2018. National Vital Statistics Reports 68: 1-11.

6. Osterman MJ, Kochanek KD, MacDorman MF, et al. (2015) Annual summary of vital statistics: 2012-2013. Pediatrics 135: 1115-1125.

7. Comstock $\mathrm{CH}$, Bronsteen RA (2014) The antenatal diagnosis of placenta accreta. BJOG 121: 171-181.

8. Sentilhes L, Kayem G, Ambroselli C, et al. (2010) Fertility and pregnancy outcomes following conservative treatment for placenta accreta. Hum Reprod 25: 2803-2810.

9. Steins Bisschop CN, Schaap TP, Vogelvang TE, et al. (2011) Invasive placentation and uterus preserving treatment modalities: $A$ systematic review. Arch Gynecol Obstet 284: 491-502.

10. Eller AG, Bennett MA, Sharshiner M, et al. (2011) Maternal morbidity in cases of placenta accreta managed by a multidisciplinary care team compared with standard obstetric care. Obstet Gynecol 117: 331-337.

11. Shamshirsaz AA, Fox KA, Salmanian B, et al. (2015) Maternal morbidity in patients with morbidly adherent placenta treated with and without a standardized multidisciplinary approach. Am J Obstet Gynecol 212: 218.e1-218.e9.

DOI: $10.36959 / 468 / 475$

Copyright: (C) 2021 Lersten IL, et al. This is an open-access article distributed under the terms of the Creative Commons Attribution License, which permits unrestricted use, distribution, and reproduction in any medium, provided the original author and source are credited. 